\title{
List of reviewers
}

JOURNAL OF ADVANCES IN LINGUISTICS (2015).

The editorial team of the journal would like to thank the following reviewers for their work in referring manuscripts during 2015.

\begin{tabular}{ll} 
Fernandes Arung & Mehrdad Vasheghani Farahani \\
Bahman Gorjian & Shaheen Akhter \\
Oshega Abang & Abdul Bari Khan \\
Somayeh Aghajani Kalkhoran & Adina Nikmawati \\
Vira - Burak & Fereidoon ---- Vahdany \\
Yana Gryshchenko & Khadija Allia Belfarhi \\
La Ino Mbangi & Miguel Ngel Gonz Lez \\
Mojtaba Eghlidi & Mahesh Bvm \\
Dr. Sarab Kadir Mugair & Dr. Vinod Bhatt \\
Asiyeh Khajeh & Vivian Meira De Oliveira \\
Metin Yurtba & Quinn Jackson \\
Roqayeh Mohammad Pour & Kais Amir Kadhim \\
Sependi Napitupulu & Mojtaba Teimourtash \\
Suswanto Ismadi Megah S. & Naseer Ahmed \\
Shahla Khalilollahi & Ayad Hammad Ali \\
Maral Nurtazina & Ramune Kasperaviciene \\
Syahfitri Purnama & Azadeh Nemati \\
Adina Nikmawati & Benson Loveday Wakama \\
Elijah Omwansa Mariera & Sahila Mustafa \\
Fadil Elmenfi & Majid Yousefi \\
Babar Mirza & Munif Zarirruddin Fikri Nordin \\
Saidna Zulfiqar Bin-Tahir & Daniele Borgogni \\
& \\
\hline &
\end{tabular}

Saloome Rostampour

Roberto Rodrigues Campos

Blessing Iyinoluwa Afolabi

Mao Zhang

Hanan A. Taqi

Nashipu Julius

Sara Montazeri

Deepak Kumar

Camille Nicole Lemieux

Masoud Rahimi Domakani

Sayyed Rashid Shah

Akintoye Oluwole Samuel

Ghaida Muttshar Abdulsahib

Masoud Bavanpouri

G Khan Ulum

K Tia Aparecida Da Silva Nunes Miranda

Mahyudin Ritonga

Hsin-I Lee

Raha Zareifard 